distribution of the amplitude and phase of the wave envelope, defined as

$\sum c_{n} \exp i\left\{\left(u_{n}-\bar{u}\right) x+\left(v_{n}-\bar{v}\right) y+\left(\sigma_{n}-\bar{\sigma}\right) t+\varepsilon_{n}\right\}$ where $\bar{u}, \bar{v}, \bar{\sigma}$ are the mean values of $u, v, \sigma$ with respect to the spectral distribution $E(u, v)$. All the results are expressed in terms of quantities which are functions of the moments

$$
m_{p q}=\int_{-\infty}^{\infty} \mathrm{d} u \int_{-\infty}^{\infty} \mathrm{d} v u p_{v} q E(u, v)
$$

of the spectral distribution. Special attention is paid to the case of a narrow spectrum, for which $E(u, v)$ is only appreciable when $u$ and $v$ lie in narrow ranges, so that the waves are approximately uniform in wave-length and direction. This situation might occur with ocean waves arising from a single distant storm area during a not too long time interval. A result of Rice ("The Mathematical Analysis of Random Noise", Bell System Technical Journal, 46 ; 1945) relating to the wave envelope of a onedimensional process can then be used to obtain an approximation to the distribution of the heights of maxima and minima of $\zeta$.

The problem of estimating $E(u, v)$ from observational data is also considered. A method of approximating to $E(u, v)$ given the values of a finite number of moments of the energy distribution for the inter. section of the surface with an arbitrary vertical plane is developed. It is shown that these moments are determined by the average numbers of zeros of $\zeta$ and its derivatives per unit distance along an arbitrary straight line, and by the mean velocities of these zeros, and hence may be estimated if values of $\zeta$ and $\partial \zeta / \partial t$ along the line are available. In the case of the sea surface it is suggested that these might be recorded by radar in an aircraft flying on a fixed course at high speed and constant altitude. No information is given about the accuracy of estimates of $E(u, v)$ made by this method. Some knowledge of the way in which the accuracy increases with the area covered by the observations for typical spectral distributions would be useful, although it might be difficult to obtain this theoretically.

A. M. WALKER

\section{VARIABLE STARS IN THE MAGELLANIC CLOUDS}

$I^{N}$ a recent paper on the spectra of some variable stars in the Magellanic Clouds (Mon. Not. Roy. Astro. Soc., 116, 5 ; 1956), M. W. Feast, of the Radcliffe Observatory, Pretoria, gives the results of a study of spectra obtained with the $f / 2$ camera of the 2-prism spectrograph $(86 \mathrm{~A} . / \mathrm{mm}$. at $\mathrm{H} \gamma)$ at the Cassegrain focus of the 74-in. Radcliffe reflector. Four variables in the Small Cloud and six in the Large Cloud were selected (another star turned out to be a galactic, foreground object) and the results are tabulated in order of increasing period. Individual velocities are not published at present, but the average velocity of stars in the Small Cloud was $133 \mathrm{~km}$. $/ \mathrm{sec}$. and in the Large Cloud $274 \mathrm{~km}$. $/ \mathrm{sec}$.; the other star, $H V$ 2882, had a low velocity. The Magellanic Cloud variables appear to be normal late $F$ and early $G$ type supergiants, and no abnormalities were noticed nor were any emission lines found to indicate that they are Population ii ( $W$ Virginis) cepheids. The results are important because the work of Gascoigne and Kron (Aust. J. Sci., Supp., 17, 23 ; 1954 ; and Pub. Astro. Soc. Pacific, 65, 32 ; 1953) led to the suggestion that the Cloud variables were in fact Population ii cepheids.

The variable $W$ Men. was discovered by Luyten (see Harvard Bulletin, $846 ; 1927$ ) and lies in the direction of the Large Magellanic Cloud. It is an $R$ CrB.-type variable, and if it is a member of the Large Magellanic Cloud it is the only star of the class known in an extragalactic system, and the only one with a known distance and absolute magnitude. Spectroscopic criteria are the only means at present for determining the absolute magnitude of this class of variable. Owing to the faintness of the star, it was necessary to use a short trail to obtain a spectrum with the $f / 2$ camera, and two spectra obtained on December 1, 1953, and February 10, 1954, were sufficiently good for certain definite conclusions to be reached. Its radial velocity is about $260 \mathrm{~km}$. $/ \mathrm{sec}$., which is in good agreement with the expected velocity for a cloud member in this position. While the spectral type is necessarily uncertain, it appears to be a supergiant of type $F 5-G 0$, most probably $F 8$ with $\mathrm{H} \gamma$ weak for the class. It may be concluded that the supergiant characteristics shown by the $R$ CrB. variables are really indicators of high luminosity in these stars. Using a modulus of $19 \cdot 2$ mag. for the clouds, given in a paper on the distances of the Magellanic Clouds by Thackeray and Wesselink in The Observatory $(75,33 ; 1955)$, an absolute magnitude at maximum for $W$ Men. of -5.4 is deduced.

\section{POLLEN IN TERRESTRIAL SOILS}

$G$ W. DIMBLEBY (New Phytol., 56, 12 ; 1957) . has pointed out that pollen analysis of terres. trial soils, as opposed to aquatic deposits, has received surprisingly little attention, especially when it is apparent that much pollen must fall on dry ground just as it does on bog surface. The assumptions that pollen falling on soils is rapidly destroyed, or removed by downwash, or so mixed and dispersed as to be valueless in studies of vegetational history, are not necessarily well founded. In fact, he has found that pollen may be present in terrestrial soils in numbers considerably exceeding those of many peat deposits. The highest frequencies, nearly 1.5 million per gm. oven-dry soil, were recorded in mineral layers, though raw humus also gave high counts. The preservation of pollen is not satisfactory in soils of which the $p \mathrm{H}$ is above 5 , but in acid soils it appears to be independent both of soil type and texture. The soil pollen content is markedly reduced by fire, and this may account for the relatively low pollen frequencies in the mineral layers of some heath soils. Acid tropical soils may contain considerable quantities of pollen, and this, together with the high values in temperate soils, suggests that the normal aerobic microbiological processes of acid soils are not so destructive of pollen as might be thought.

The distribution curve of each species of pollen through the profile assumes a characteristic form according to the length of time the pollen has been in the soil, owing to the gradual downward movement under the influence of percolating water. This movement is normally a very slow process. From the distribution patterns it is possible to outline the broad features of past vegetation changes, but interpretation is always comparative, and independent time fixes must be obtained for absolute dating. 